\title{
Análisis bibliométrico y \\ cienciométrico de la producción \\ científica de Perú y Ecuador desde \\ Web of Science (2009-2018)
}

\section{(1) Cesar H. Limaymanta}

Universidad Nacional Mayor de San Marcos. Escuela de Bibliotecología y Ciencias de la Información. Universidad Peruana de Ciencias Aplicadas. Área de Ciencias, Perú I climaymantaa@unmsm.edu.pe / pcmaclim@upc.edu.pe /

https://orcid.org/oooo-0002-8797-4275

\section{Hilda Zulueta-Rafael}

Universidad Nacional Mayor de San Marcos. Escuela de Bibliotecología y Ciencias de la Información, Perú

| hilda.zulueta@unmsm.edu.pe / https://orcid.org/oooo-0003-2368-9085

\section{Cristina Restrepo-Arango}

Universidad de Córdoba. División de Bibliotecas y Recursos Educativos, Colombia | cristinarestrepo@correo.unicordoba.edu.co / https://orcid.org/oooo-0003-4275-4102

\section{Patricio Álvarez-Muñoz}

Facultad de Ciencias Administrativas, Universidad Estatal de Milagro, Ecuador | palvarezm@unemi.edu.ec / https://orcid.org/oooo-0002-9754-8050

\section{Resumen}

Este artículo compara la producción científica de Perú y Ecuador entre 2009 y 2018. Se recuperaron documentos con al menos una afiliación peruana o ecuatoriana desde el Science Citation Index Expanded, Social Sciences Citation Index, Arts er Humanities Citation Index y Emerging Sources Citation Index de la base de datos Web of Science. Se evaluaron la tendencia y el crecimiento exponencial de la producción científica, se identificaron instituciones y áreas temáticas más productivas. Se estudió la estructura intelectual de la producción científica peruana y ecuatoriana mediante el análisis de acoplamiento bibliográfico de autores (AABA) y el análisis de cocitación de autores (ACA). Los resultados muestran que Perú tiene mayor producción acumulada que Ecuador. Sin embargo, Ecuador tiene mayor producción en los tres últimos años, incluso con pronóstico de seguir produciendo más que Perú. Las instituciones más productivas en ambos países son las universidades, y las categorías temáticas más productivas para Perú son salud ocupacional y ambiental y, para Ecuador son investigación educativa y ciencias ambientales.

Palabras clave Bibliometría Universidades VOSviewer Acoplamiento Bibliográfico Cocitación 


\section{Bibliometric and scientometric analysis of the scientific production of Peru and Ecuador from Web of Science (2009-2018)}

\section{Abstract}

This article compares the scientific production of Peru and Ecuador between 2009 and 2018. Documents with at least one Peruvian or Ecuadorian affiliation were retrieved from the Science Citation Index Expanded, Social Sciences Citation Index, Arts $\mathcal{E}$ Humanities Citation Index and Emerging Sources Citation Index of the Web of Science database. The trend and exponential growth of scientific production were evaluated, more productive institutions and thematic areas were identified. The intellectual structure of the Peruvian and Ecuadorian scientific production was studied by means of the authors bibliographic-coupling analysis (ABCA) and the authors co-citation analysis (ACA). The results show that Peru has greater accumulated production than Ecuador. However, Ecuador has more production in the last three years, even with a forecast to continue producing more than Peru. The most productive institutions in both countries are the universities, and the most productive subject categories for Peru are occupational and environmental health, and for Ecuador they are educational research and environmental sciences.

Artículo recibido: 15-04-2020. Aceptado: 23-10-2020

\section{Introducción}

La evaluación de la investigación se viene aplicando de manera progresiva desde hace varias décadas en la academia. Sus resultados son un insumo de gran valor para la optimización de los recursos públicos (Moed, 2009). Algunos autores proponen dos tipos de indicadores para evaluar los sistemas de ciencia y tecnología, (i) los indicadores de insumo (input) y (ii) los indicadores de producción científica (output). El primero, define el comportamiento de países o regiones en cuanto al aspecto económico y de talento humano, así como proporcionan información para desarrollar las investigaciones. El segundo, gira en torno a la evaluación de las investigaciones mediante la publicación en revistas científicas (Jaramillo-Salazar, Botiva y Zambrano, 2004).

La producción científica es el resultado de un proceso de investigación que se lleva a cabo en laboratorios, industrias, universidades, entre otros lugares; para publicar los resultados en la forma de productos como libros, artículos, ponencias, capítulos de libros, etc. (Carvajal-Tapia y Carvajal-Rodríguez, 2019). El análisis de la producción científica permite conocer las tendencias de publicación por autores, temáticas, instituciones, países y regiones, entre otros. Producto de esto, identifica la capacidad institucional y nacional para producir ciencia (Pouris, 2012). Las universidades son una de las instituciones que aportan al avance científico de un país. Por tal motivo, en los últimos diez años se han implementado medidas que dirigen a las universidades a priorizar, no solo en educación sino también en investigación. En el caso peruano se promulgó la Ley $\mathrm{N}^{\circ} 30220$ el año 2014, cuyo objeto es promover la calidad en las universidades como agentes propiciadores del desarrollo social, cultural y científico. A partir de la promulgación de esta ley se creó la Superintendencia Nacional de Educación Superior Universitaria (SUNEDU), ente responsable del licenciamiento para el servicio educativo superior universitario. El licenciamiento es el procedimiento que tiene como objetivo verificar el cumplimiento de las condiciones básicas de calidad para ofrecer el servicio educativo superior universitario y autorizar su funcionamiento (Perú. Congreso de la República, 2014). Esta ley ha contribuido para que las universidades peruanas realicen inversiones en la generación del conocimiento y 
promuevan esfuerzos que incentiven la publicación de los resultados de investigación y así incrementar la visibilidad y el impacto. Además, mediante el artículo 86 de la mencionada ley permitió la creación de una nueva categoría de "docente investigador" llamado investigador en el Registro Nacional de Ciencia, Tecnología y de Innovación Tecnológica (Renacyt) que designa a los catedráticos con excelencia académica, cuyos beneficios son tener carga lectiva de un curso por año y bonificación adicional del $50 \%$ de sus haberes totales por un período de nueve meses, de abril a diciembre.

Ecuador ha implementado medidas para el desarrollo de la investigación científica. La promulgación de la Ley Orgánica de Educación Superior (LOES) en el año 2010 junto con su reglamento y la implementación del nuevo escalafón del docente e investigador, incrementaron el crecimiento de la investigación científica en las universidades ecuatorianas. También aumentó el número de doctores porque la LOES estipula que todos los docentes universitarios deben tener como mínimo una maestría y alrededor del 70\% de ellos un doctorado. Asimismo, el gobierno impulsó mecanismos públicos que facilitaron el acceso a las fuentes de información científica como bibliografía actualizada y acceso a bases de datos bibliográficas (Álvarez-Muñoz y Pérez-Montoro, 2015). La LOES creó el Consejo de Evaluación, Acreditación y Aseguramiento de la Calidad de la Educación Superior (CEAACES), hoy en día denominado Consejo de Aseguramiento de la Calidad de la Educación Superior (CASES) que supervisa, evalúa y motiva a las universidades a otorgar mayor importancia a la investigación (CEAACES, 2018). Adicionalmente, un factor clave para el crecimiento de la productividad ecuatoriana lo tuvo el programa Prometeo "Viejos Sabios", el cual contribuyó al aumento de la producción y colaboración científica internacional. El programa en mención comenzó el año 2010 e incorporó científicos reconocidos del extranjero para vincularlos con instituciones nacionales, con el objetivo de potenciar el desarrollo de la investigación y crear un ecosistema científico en ese país. El número de expertos extranjeros que arribaron a Ecuador alcanzó su punto máximo en los años 2013 y 2014 con 253 y 468 personas respectivamente (Castillo y Powell, 2019). Cabe resaltar que a partir del año 2014 Ecuador refleja un crecimiento continuo de publicaciones indexadas en Web of Science (WoS en adelante). Otro aspecto que debe destacarse es que antes del 2008 no hubo una política clara de investigación ni incentivos para los científicos, además a esto se le suma el crecimiento desregularizado de centros privados en décadas anteriores que es una de las causas de la escasa producción científica en casi todos los países de Latinoamérica (Álvarez-Muñoz y Pérez-Montoro, 2015).

Las medidas normativas y de creación de instituciones que fueron adoptadas por Perú y Ecuador para fomentar la investigación y la educación superior tienen como eje central el desarrollo económico, ya que tanto la educación como la investigación son los impulsores del desarrollo económico. Es importante considerar que el desarrollo económico se basa en la generación de conocimiento, ambos conceptos se empezaron a discutir a partir de la década del 60. Varias instituciones internacionales como la ONU (Primera conferencia de la Organización de las Naciones Unidas sobre la aplicación de CyT al desarrollo, en beneficio de las regiones menos avanzadas), la OCDE, la UNESCO, el International Development Research Center (IDRC), el Banco Interamericano de Desarrollo (BID) y la Organización de Estados Americanos (OEA), fomentaron varias iniciativas para la creación de tejidos científicos que sustenten investigaciones relevantes en cada país. Todo esto, con el fin de contribuir al desarrollo de la ciencia, y así fomentar la creación de instituciones responsables del tema en diversos países de Latinoamérica (Jaramillo-Salazar, Botiva y Zambrano, 2004). Por ejemplo, México fue el primero en adoptar estas iniciativas con la creación del Instituto Nacional de la Investigación Científica (INIC) en 1950, luego Brasil con la creación del Conselho Nacional de Desenvolvimento Científico e Tecnológico (CNPq) en el año 1951 y por último Argentina en 1958 creó el Consejo Nacional de Investigadores Científicas y Técnicas (CONICET). Por ende, no es de extrañarse que precisamente 
estos países son los que lideran la evolución de la producción científica en Latinoamérica. No obstante, en los últimos años ha mejorado la evolución de la producción científica, producto de mejores políticas de inversión social, políticas de generación de conocimiento, fortalecimiento del ecosistema de investigación, y la preocupación de tener presencia en el mapa de la ciencia regional y mundial producto de la mayor cantidad de personal con títulos de $\mathrm{PhD}$ que presionan por un mejor sistema de educación superior.

En ese contexto, este artículo pretende ser un instrumento de apoyo para la toma de decisiones de autoridades políticas y académicas tanto de Perú y Ecuador, además llenar un vacío en la investigación cienciométrica, pues no se han realizado estudios de la producción científica de Ecuador y Perú en el Science Citation Index de WoS (Sisa et al., 2011; Huamaní y Mayta-Tristán, 2010). El objetivo general es analizar la producción científica de Perú y de Ecuador en las bases de datos Science Citation Index Expanded (SCIE), Social Sciences Citation Index (SSCI), Arts er Humanities Citation Index (A\&HCI) y Emerging Sources citation Index (ESCI) pertenecientes a WoS Core Collection periodo 2009-2018. Países latinoamericanos y fronterizos, comparten la misma lengua (español) y presentan una producción científica en crecimiento paulatino. Los objetivos específicos son: (1) Evaluar la tendencia y el crecimiento de la producción científica de Perú y Ecuador entre los años de 2009 a 2018; (2) Identificar las instituciones más productivas, áreas temáticas y tipos de documentos producidos para Perú y Ecuador; y (3) Identificar la estructura intelectual en publicaciones con afiliación peruana y ecuatoriana según la cocitación y el acoplamiento bibliográfico de los autores.

\section{Estado del arte de estudios bibliométricos de Perú y/o Ecuador}

El desarrollo de la bibliometría ha permitido medir y comparar los esfuerzos de instituciones, tanto públicas como privadas en los procesos de generación de ciencia. Estos tipos de estudios permiten conocer el estado de las actividades de investigación y a su vez muestran el camino para mejorar su calidad. Su importancia es fundamental para países en vías de desarrollo con sistemas frágiles de ciencia y tecnología como son Ecuador y Perú (Bornmann y Daniel, 2008; Lascurain-Sánchez, 2006; Leydesdorff, 2008). En ese sentido, se buscaron estudios bibliométricos sobre la producción científica peruana y ecuatoriana en las bases de datos WoS, Scopus y Scielo. Se encontraron trabajos que analizaron la producción científica peruana en determinados temas como tecnologías de la información (Asmat-Vega et al., 2019), disparidades de género entre médicos peruanos (Amaya, Mougenot y Herrera-Añazco, 2019), salud mental (Ocaña-Fernández et al., 2019), bibliometría (Urbizagástegui-Alvarado, 2014). También se encontraron trabajos de análisis bibliométricos a universidades (Roman-Gonzalez, Ciriaco-Susanibar y Vargas-Cuentas, 2019) o a revistas (Romaní y Cabezas, 2018). Para Ecuador, existen estudios sobre la producción científica de dicho país en el contexto andino (Álvarez-Muñoz y Pérez-Montoro, 2015) y el impacto de la colaboración internacional (Castillo y Powell, 2019). Existen pocos estudios bibliométricos en temas específicos; por ejemplo, Sisa et al. (2011) estudiaron la producción científica ecuatoriana en ciencias de la Salud. Por lo tanto, luego de esta revisión no se encontraron trabajos que analicen comparativamente la producción científica de Perú y Ecuador como la que se pretende realizar en este trabajo.

\section{Metodología}

Para reunir la producción científica con afiliación peruana y ecuatoriana se usaron las bases de datos SCIE, SSCI, AeHCI y ESCI de WoS Core Collection. Estas bases de datos se seleccionaron porque facilitan la recolección de datos para realizar estudios bibliométricos y cienciométricos. Se considera una plataforma de bases de datos pionera 
que contiene publicaciones de calidad, de revistas seleccionadas por los editores internos expertos de $W o S$ con rigor editorial tomando en cuenta las mejores prácticas.

La recuperación de la información se hizo en noviembre del 2019 con la ecuación de búsqueda avanzada: $\mathrm{CU}=$ Perú y $\mathrm{CU}=$ Ecuador, de manera independiente y se filtró la información teniendo en cuenta el periodo de tiempo de 2009 a 2018. Se tomó como año de inicio el 2009 porque se pretende analizar el comportamiento de la producción científica de la última década en ambos países. WoS admite descargas de varios formatos de archivo y se usó en este estudio el delimitado por tabulador (Win). Para cada documento se obtuvo el registro completo, incluidas las referencias citadas. Para analizar la tendencia de la producción científica se aplicó el análisis de regresión no lineal con el programa Minitab v18, siendo el cuadrático (ecuación 1) el mejor modelo por tener un mayor índice de bondad de ajuste (coeficiente de determinación $\mathrm{R}^{2}$ ) que estimó los modelos de tendencia para ambos países. Donde $y$ es la producción estimada en el tiempo t; con coeficientes $b_{0}, b_{1}$ y $b_{2}$. Para analizar la producción científica acumulada de cada país se empleó el modelo exponencial de Price (ecuación 2), donde $\mathrm{N}$ es la producción acumulada, $\mathrm{N}_{\mathrm{o}}$ es la producción medida en el tiempo $t=0$ y b es la constante que relaciona la velocidad del crecimiento con el tamaño acumulado de la producción científica (Price, 1963). Otros estudios también utilizaron este modelo (Bazm, Bazm y Sardari, 2019; Roy, 2019)

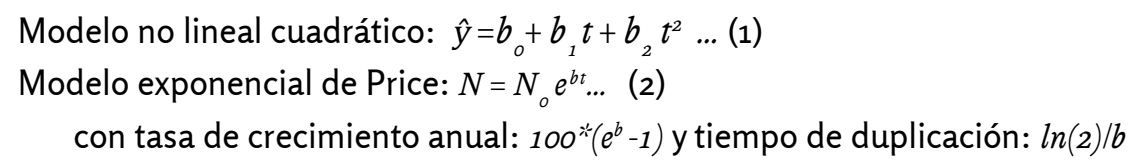

Para analizar las instituciones más productivas, las temáticas y los tipos de documentos se extrajeron los datos de la plataforma WoS hacia el Microsoft Excel 2016. Para analizar el acoplamiento bibliográfico y la cocitación se usó el programa VOSviewer v1.6.13 previa construcción de tesauros de normalización para cada mapa. Con este programa se obtuvieron los mapas de visualización de redes bibliométricas basados en distancia (Van Eck y Waltman, 2014). En la elaboración de estas redes se empleó el método fractional counting el cual asigna el mismo peso a cada acción y es recomendado para este tipo de análisis (Perianes-Rodríguez, Waltman y Van Eck, 2016; Van Eck y Waltman, 2014). Las cinco fases de la metodología que se usaron para la construcción de las visualizaciones científicas fueron: (1) recopilación de información, (2) definición de unidades de análisis, (3) definición de unidades de medida, (4) reducción de dimensionalidad y distribución de visualizaciones y (5) análisis e interpretación de la visualización (Vargas-Quesada y De-Moya-Anegón, 2007).

La red bibliométrica está compuesta por nodos (unidades de análisis) y vínculos (relaciones). Para este trabajo los nodos son: i) los autores citantes y ii) los autores referenciados o citados; y como vínculos se usaron: i) acoplamiento bibliográfico y ii) cocitación respectivamente. El acoplamiento bibliográfico se da cuando dos publicaciones A y B citan en común a una tercera publicación $C_{i}$ (Kessler, 1963). En este caso A y B están acoplados bibliográficamente y la frecuencia de citas comunes $\mathrm{C}_{1} ; \mathrm{C}_{2} ; \mathrm{C}_{3} \ldots$ indica la fuerza de acoplamiento entre $\mathrm{A}$ y $\mathrm{B}$. Este artículo desarrolla el análisis del acoplamiento bibliográfico de autores (AABA) como un método para mapear autores activos en los documentos analizados (Zhao y Strotmann, 2008), obteniendo así una imagen real del estado actual de sus investigaciones. Por su parte, la cocitación es una medida de similitud o relación entre unidades de análisis que hace uso de relaciones de citas directas y se define como la frecuencia con la que dos documentos son citados conjuntamente por otros documentos (Wang et al., 2016). Si al menos un documento A cita dos documentos en común B y C, 
se dice que estos documentos son cocitados. El análisis de cocitación de autores (ACA) como una técnica para identificar la estructura intelectual de las disciplinas científicas se da cuando dos ítems (autores B y C en este caso) son citados por un tercer ítem (autor A) en su trabajo propio (White y Griffith, 1981; Zhao y Strotmann, 2008). En este artículo se presentan el AABA para identificar a los autores activos y el estado actual de las investigaciones; $y$ el ACA para identificar la estructura de las influencias intelectuales (autores que han influido en los autores activos). Ambos análisis otorgar una imagen clara de la estructura intelectual de la producción científica peruana y ecuatoriana.

\section{Resultados}

\subsection{Tendencia y crecimiento acumulado de la producción científica anual}

La producción científica peruana indexada en WoS suma un total de 16.328 registros. Durante los años 2009-2018 la producción científica ha ido aumentando; en el 2009 se registraron 885 publicaciones, en el 2018 el número aumentó a 2.995 publicaciones. Por su parte, Ecuador registra un total de 13.787 publicaciones; en el 2009 contaba con 417 publicaciones, mientras que en el 2018 el número creció a 3.580, es decir, 585 publicaciones más que Perú en ese año (Gráfico 1). Asimismo, se observa que en el año 2016 Ecuador logra superar a Perú en su producción científica. Uno de las causas de estos resultados se debe a la inversión en investigación y desarrollo ( $\mathrm{I}+\mathrm{D})$ que realizaron ambos países, ya que según datos disponibles del Banco Mundial (2019) Perú invirtió en I+D en el año 2014 un $0,108 \%$ del producto interior bruto (PIB o también denominado PBI); Ecuador, en ese mismo año un $0,443 \%$ (cuatro veces lo invertido por Perú). La inversión en $\mathrm{I}+\mathrm{D}$ que se realiza en un determinado año se ve reflejada en producción científica después de uno o dos años aproximadamente.

Mediante el análisis de regresión no lineal se estimaron los mejores modelos de tendencia para ambos países; el mejor modelo es el cuadrático, ecuación (3) con $\mathrm{R}^{2}=95,51 \%$ y (4) con $\mathrm{R}^{2}=97,45 \%$. Asimismo, se realizó un pronóstico de la producción científica para el 2019 y 2020 que se muestran con color verde (Gráfico 1). De seguir la misma tendencia de crecimiento, Ecuador producirá más que Perú en los próximos años.

$$
\begin{aligned}
& * * y(\text { Perú })=925-95,2 t+31,97 t^{2} \ldots \text { (3) } \\
& * * y(\text { Ecuador })=693-321 t+63,63 t^{2} \ldots \text { (4) }
\end{aligned}
$$

El Gráfico 2 muestra el análisis de la producción científica acumulada mediante el modelo de Price, se estimaron los modelos para Perú $\left(R^{2}=96,78 \%\right)$ como para Ecuador $\left(\mathrm{R}^{2}=99,47 \%\right)$. Estos modelos muestran que la productividad científica de ambos países sigue un crecimiento exponencial. La tasa de crecimiento anual de Perú es $35,1 \%$ y se espera que el tiempo de duplicación de su producción científica sea de 2,3 años. Para Ecuador es de 45,6\% anual y se estima que el tiempo de duplicación de su producción científica sea 1,8 años. Ecuador muestra una mayor tasa de crecimiento anual y un menor tiempo de duplicación que Perú. 
Gráfico 1. Distribución anual de la producción científica de Perú y Ecuador (20092018) con pronósticos para 2019 y 2020

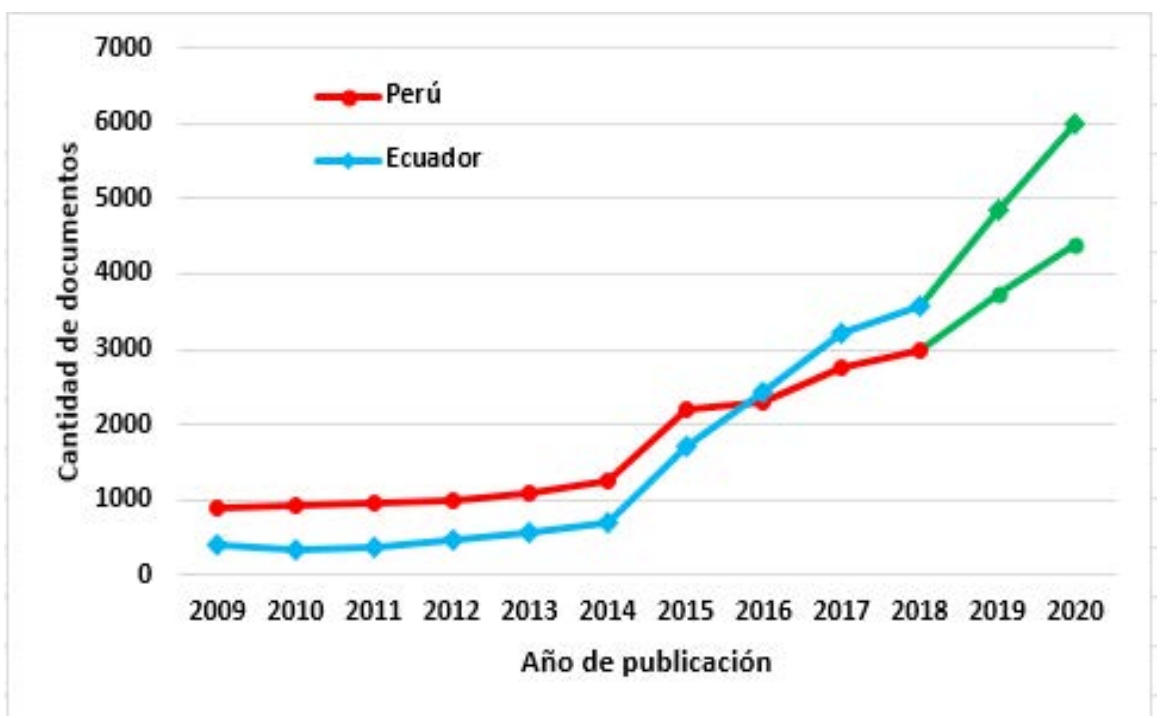

Gráfico 2. Modelo exponencial del crecimiento acumulado de la producción científica de Perú y Ecuador

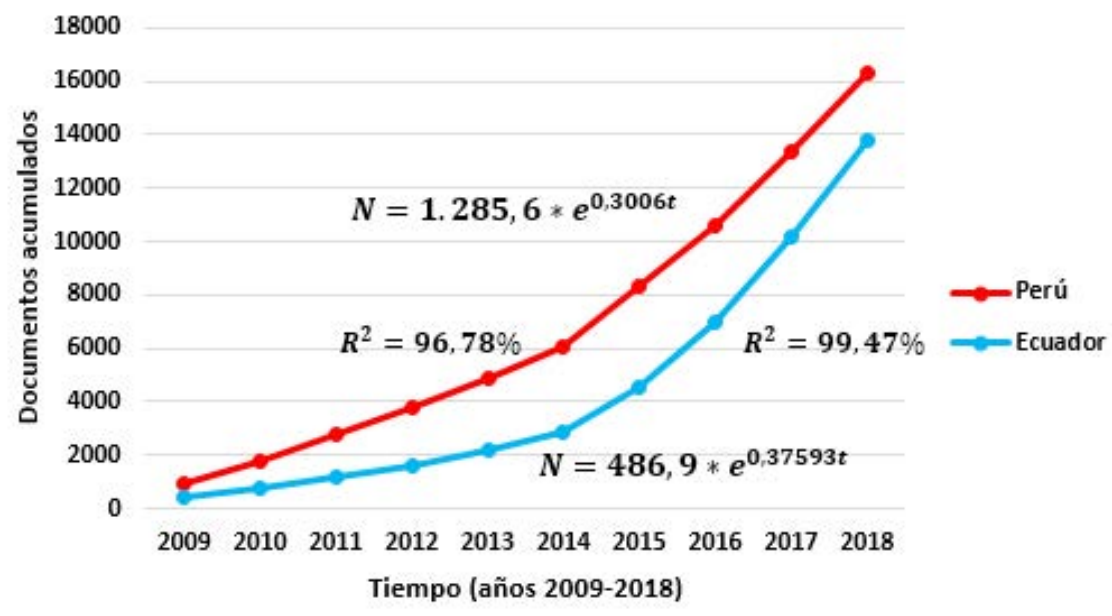

\subsection{Instituciones más productivas, áreas temáticas y tipos de documentos}

Si bien es cierto la educación en todos sus niveles es un factor clave para la formación de capital humano y el desarrollo de una nación, la educación superior destaca por tener un vínculo más directo con el sector productivo (SUNEDU, 2018). Las universidades al poner en práctica actividades de investigación e innovación ayudan a ampliar la frontera de producción y mejorar la eficiencia en la asignación de recursos; de tal forma que produzca un incremento de la riqueza en la sociedad (Yasar-Akcali y Sismanoglu, 2015). A partir del 2016, gracias a las acciones del Estado peruano, como el licenciamiento y la acreditación, las universidades buscaron estimular las actividades en torno a la investigación. Asimismo, otras iniciativas del gobierno fueron 
el financiamiento de proyectos de investigación, la mejora de la infraestructura y la creación de laboratorios en las universidades, las becas de maestría y doctorado a través de programas nacionales e internacionales, y el fomento de la movilidad de científicos jóvenes (postdocs) y seniors al país (Gago Medina, 2016). Producto de esto, el top cinco de las instituciones peruanas más productivas en WoS durante la última década son precisamente universidades: Universidad Peruana Cayetano Heredia (UPCH), Pontificia Universidad Católica del Perú (PUCP), Universidad Nacional Agraria La Molina (UNALM), Universidad Nacional Mayor de San Marcos (UNMSM) y Universidad Peruana de Ciencias Aplicadas (UPC) (Gráfico 3).

Gráfico 3. Top 5 de las instituciones peruanas más productivas (2009-2018)

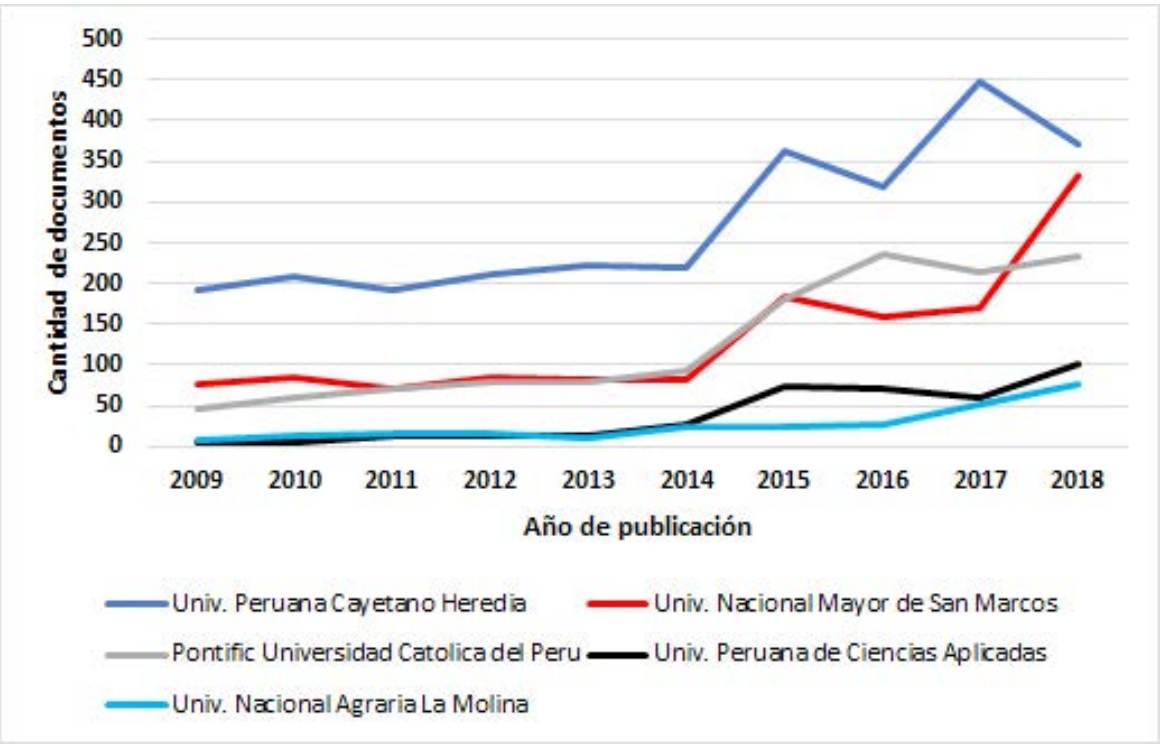

La educación superior en el contexto ecuatoriano tuvo un cambio positivo tras la promulgación de la nueva constitución en el 2008. La entrada en vigencia de la nueva LOES en 2010 propició como resultado el aumento de doctorados en la plana docente. La calidad en las instituciones de educación superior (IES), a partir de la evaluación y la acreditación de las universidades y escuelas politécnicas se dio a través del CEAACES, proceso que calificó a las IES en cuatro categorías (A, B, C, y D) según el cumplimiento de estándares de calidad requeridos. La Secretaría Nacional de Educación Superior, Ciencia, Tecnología e Innovación (SENESCYT) encargada de estipular las políticas estatales de educación superior, fomenta la investigación gracias a su programa de becas y el financiamiento de proyectos de investigación (Rivera García, Espinosa Manfugás y Valdés Bencomo, 2017).

La reforma de la educación superior ecuatoriana que se inició en el 2008 fue la causa del notable desarrollo de la actividad científica, la investigación y la innovación en las universidades y escuelas politécnicas del país. Esto se evidencia en el crecimiento de la producción científica de las IES más productivas, puesto que es aún mayor al crecimiento presentado por las universidades peruanas. Las universidades con mayor producción en Ecuador son: Universidad San Francisco de Quito, Universidad de los Andes, Universidad de Cuenca, Pontificia Universidad Católica del Ecuador y Universidad Técnica Particular de Loja (Gráfico 4). 
Gráfico 4. Top 5 de las instituciones ecuatorianas más productivas (2009-2018)

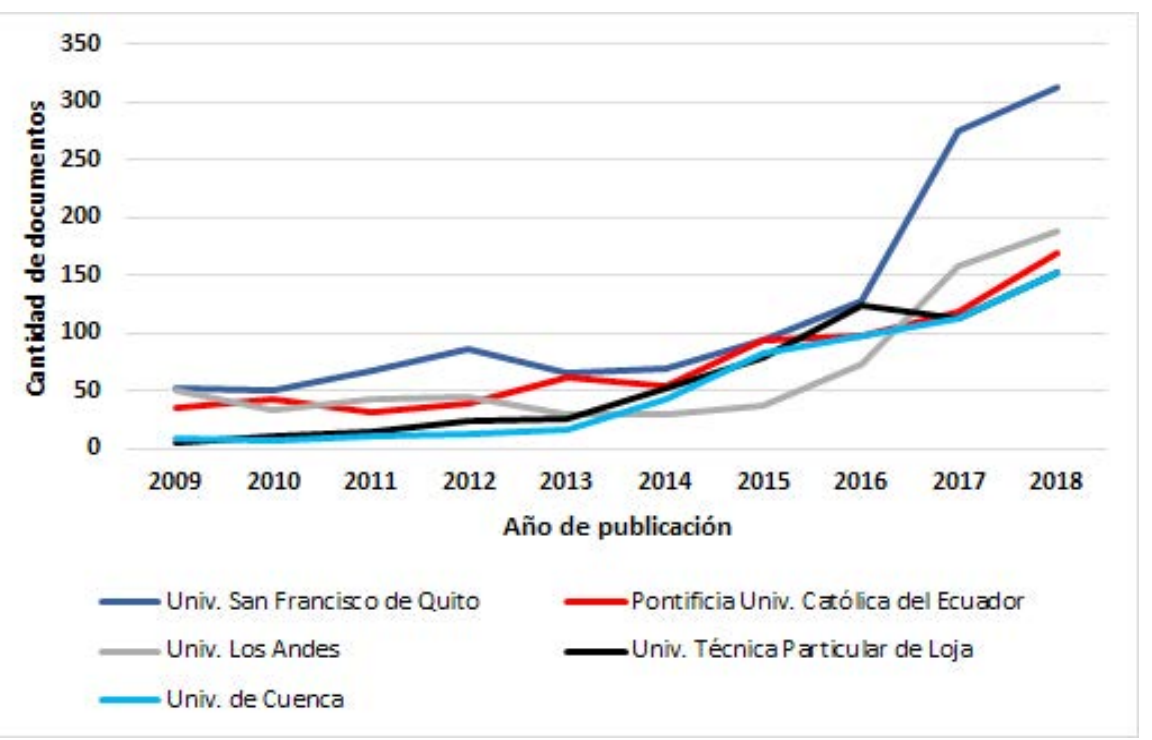

Uno de los intereses comunes de las instituciones nacionales que financian proyectos de investigación, tales como el CONCYTEC de Perú y la SENESCYT de Ecuador es conocer las áreas temáticas que tienen mayor producción científica para tomar decisiones y conocer el perfil investigador de una nación. La Tabla 1 presenta la distribución del top 10 de las disciplinas de mayor producción científica, según el área temática por cada país. Para el caso peruano, las 10 primeras disciplinas pertenecen al campo de las ciencias médicas, las ciencias económicas y las ciencias naturales. Por su parte, Ecuador presenta mayor productividad en la investigación educativa, las ciencias ambientales, la ingeniería y las ciencias sociales. Cabe resaltar que debido a la multi asignación de algunas revistas a categorías temáticas que realiza WoS existen documentos de dichas revistas que están clasificadas en más de un área temática.

Tabla 1. Distribución del top 10 de las áreas temáticas con mayor producción

\begin{tabular}{|c|l|c|c|l|c|c|}
\hline \multirow{2}{*}{$\mathbf{N}^{\circ}$} & \multicolumn{5}{|c|}{ Perú É } & \multicolumn{5}{c|}{ Ecuador } \\
\hline $\mathbf{1}$ & $\begin{array}{l}\text { Aalud ocupacional y } \\
\text { ambiental }\end{array}$ & 1.156 & $10 \%$ & Investigación educativa & 1.348 & $10 \%$ \\
\hline $\mathbf{2}$ & Enfermedades infecciosas & 1.115 & $7 \%$ & $\begin{array}{l}\text { Ecología de ciencias } \\
\text { ambientales }\end{array}$ & 1.172 & $9 \%$ \\
\hline $\mathbf{3}$ & Medicina tropical & 1.087 & $7 \%$ & Ingeniería & 1.061 & $8 \%$ \\
\hline $\mathbf{4}$ & Ciencias multidisciplinarias & 752 & $5 \%$ & Ciencias sociales & 1.004 & $7 \%$ \\
\hline $\mathbf{5}$ & Medicina general interna & 671 & $4 \%$ & Física & 769 & $6 \%$ \\
\hline $\mathbf{6}$ & Oncología & 643 & $4 \%$ & Ciencia y tecnología & 598 & $4 \%$ \\
\hline $\mathbf{7}$ & Inmunología & 545 & $3 \%$ & Química & 514 & $4 \%$ \\
\hline $\mathbf{8}$ & Ciencias ambientales & 466 & $3 \%$ & Ciencias de las plantas & 504 & $4 \%$ \\
\hline $\mathbf{9}$ & Ciencias de las plantas & 440 & $3 \%$ & $\begin{array}{l}\text { Salud ocupacional y } \\
\text { ambiental }\end{array}$ & 494 & $4 \%$ \\
\hline $\mathbf{1 0}$ & Economía & 430 & $3 \%$ & Zoología & 479 & $3 \%$ \\
\hline
\end{tabular}


En cuanto al tipo de documento, se observa que el artículo científico es el tipo más frecuente $72 \%$ y $86,1 \%$ para Perú y Ecuador respectivamente, seguido de resúmenes de reunión ( $15 \%$ y $5,9 \%)$, y revisiones (3,9\% y $3,5 \%)$. En la categoría "Otros" se encuentran los tipos de documentos con menor frecuencia de publicación, tales como las reseñas bibliográficas (book review), actas de conferencias (proceedings paper), correcciones (correction), capítulos de libros (book chapter), poemas (poetry), etc. que conforman el 2,5\% y $1,4 \%$ de toda la producción científica de Perú y Ecuador respectivamente como se muestra en el Gráfico 5.

Gráfico 5. Distribución de registros según tipo de documento por país

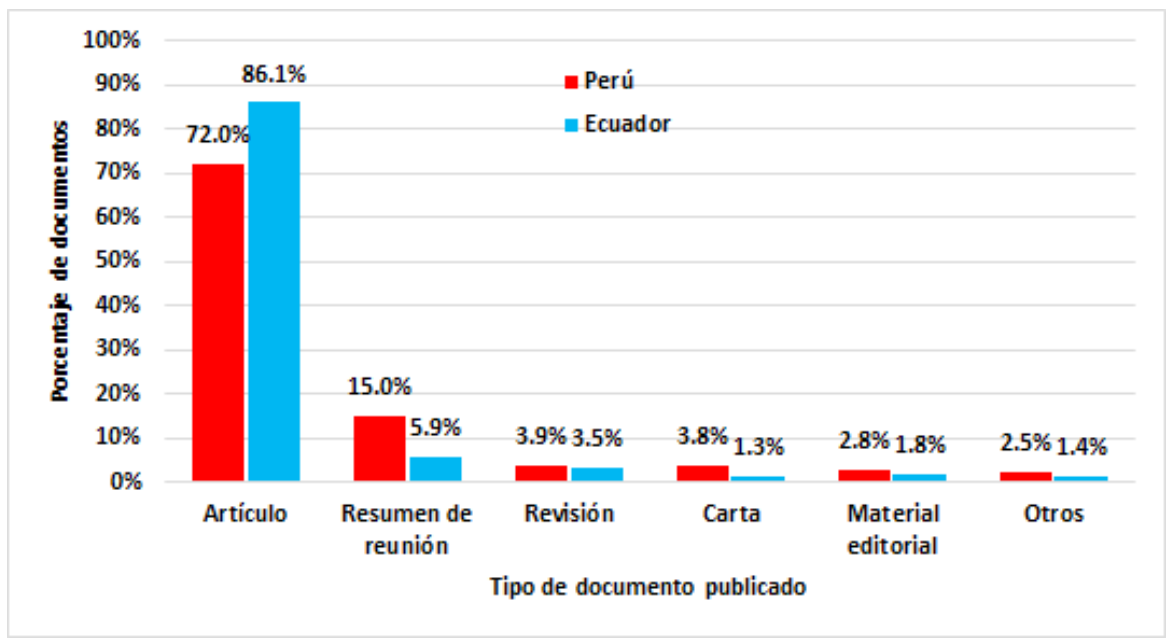

\subsection{Estructura intelectual según acoplamiento bibliográfico y cocitación de autores}

3.3.1. Acoplamiento bibliográfico de autores en documentos con al menos una afiliación peruana

Para identificar la estructura del estado actual de los principales autores en documentos con al menos una afiliación peruana se realiza el AABA (Gráfico 6); cada círculo (nodo) representa un investigador. El tamaño del círculo representa la fuerza de acoplamiento bibliográfico de los investigadores, tal es el caso de Gilman, R.H. y Miranda, J.J. con 10.621,18 y 5.181,17 de índice de fortaleza respectivamente. De acuerdo con el acoplamiento bibliográfico, cuanto más cerca se encuentran dos investigadores en la visualización, mayor será la relación entre ellos. Es decir, investigadores que se ubican cerca el uno del otro, tienden a citar las mismas publicaciones; por ejemplo, Kochel, T. y Halsey, E. Los colores indican agrupaciones de investigadores que están fuertemente relacionados entre sí según la fuerza de acoplamiento bibliográfico de autores. 
Gráfico 6. Visualización de una red de acoplamiento bibliográfico de autores que publicaron en documentos con al menos una afiliación peruana

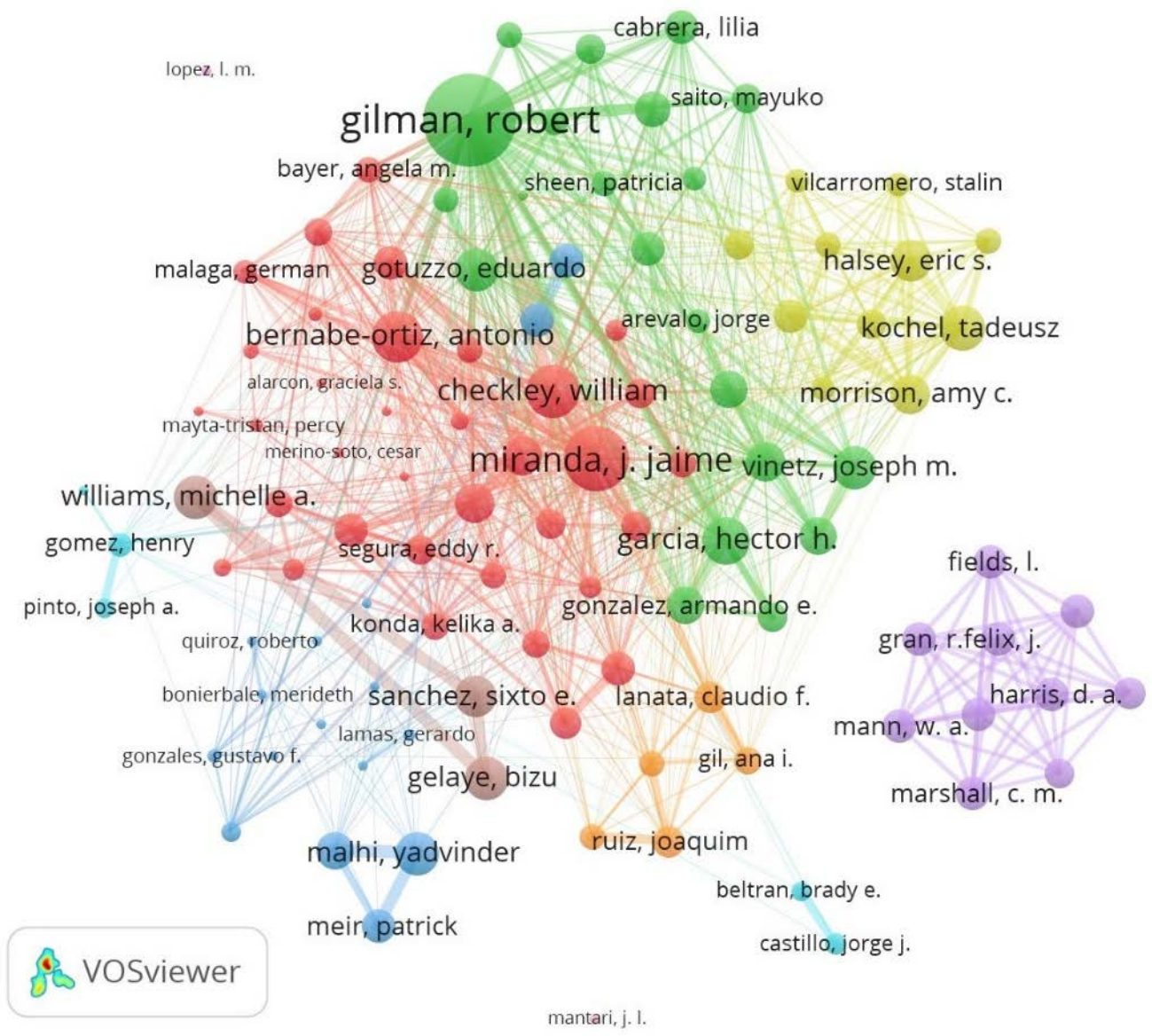

Nota: Se usaron documentos con 500 autores como máximo por documento, autores con 30 documentos como mínimo. De los 69.482 autores, 108 cumplen el umbral; atracción: 1; repulsión: -5; resolución de agrupamiento: 0,5

En la Tabla 2 se aprecia en forma descendente los autores de los cinco principales clústeres con mayor fuerza de enlace de acoplamiento bibliográfico. Cada autor con su respectiva institución de afiliación, sus principales categorías de investigación, la cantidad de documentos, y la fuerza de enlace total. Así, Gilman, R. que se encuentra en el clústerverde, es el autor con mayor fuerza de acoplamiento bibliográfico afiliado a la Johns Hopkins Bloomberg School of Public Health e investiga temas relacionados a salud pública y medicina tropical. En esa misma línea están los autores que se encuentran en los clústeres rojo, verde y amarillo. En el clúster azul se encuentran autores que investigan temas relacionados a ecología y medio ambiente; mientras que en el clúster morado temas de física. Entre los autores representativos peruanos se encuentran Miranda, J., Bernabe-Ortiz, A., García, H. y Gotuzzo, E. que investigan temas relacionados a medicina, salud pública, aunque este último específicamente a las enfermedades infecciosas. Gran parte de estas áreas temáticas que investigan los principales autores coinciden con los resultados presentados en la Tabla 1. 
Tabla 2. Autores de los cinco clústeres con mayor fuerza de acoplamiento bibliográfico en documentos con afiliación peruana

\begin{tabular}{|c|c|c|c|c|c|c|}
\hline $\mathrm{N}^{\circ}$ & Autor & Cluster & Institución/país & $\begin{array}{l}\text { Principales áreas temáticas/ } \\
\text { categorías de WoS }\end{array}$ & ND & FET \\
\hline 1 & $\begin{array}{l}\text { Miranda, J. } \\
\text { Jaime }\end{array}$ & \multirow{3}{*}{ Rojo } & $\begin{array}{l}\text { Universidad Peruana } \\
\text { Cayetano Heredia/Perú }\end{array}$ & $\begin{array}{l}\text { Salud ocupacional ambiental } \\
\text { pública/Sistemas cardiovasculares } \\
\text { cardiacos }\end{array}$ & 173 & $5.181,17$ \\
\hline 2 & $\begin{array}{l}\text { Checkley, } \\
\text { William }\end{array}$ & & $\begin{array}{l}\text { Johns Hopkins University/ } \\
\text { USA }\end{array}$ & $\begin{array}{l}\text { Sistema respiratorio/Medicina de } \\
\text { terapia intensiva }\end{array}$ & 152 & $3.463,91$ \\
\hline 3 & $\begin{array}{l}\text { Bernabe- } \\
\text { Ortiz, Antonio }\end{array}$ & & $\begin{array}{l}\text { Universidad Peruana } \\
\text { Cayetano Heredia/Perú }\end{array}$ & $\begin{array}{l}\text { Salud ocupacional ambiental } \\
\text { pública/Ciencias multidisciplinarias }\end{array}$ & 116 & $3.258,08$ \\
\hline 4 & $\begin{array}{l}\text { Gilman, } \\
\text { Robert }\end{array}$ & \multirow{3}{*}{ Verde } & $\begin{array}{l}\text { Johns Hopkins Bloomberg } \\
\text { School of Public Health/USA }\end{array}$ & $\begin{array}{l}\text { Salud ocupacional ambiental } \\
\text { pública/Medicina Tropical }\end{array}$ & 456 & $10.621,18$ \\
\hline 5 & $\begin{array}{l}\text { Garcia, } \\
\text { Hector H. }\end{array}$ & & $\begin{array}{l}\text { Universidad Peruana } \\
\text { Cayetano Heredia/Perú }\end{array}$ & $\begin{array}{l}\text { Medicina Tropical/Salud } \\
\text { ocupacional ambiental pública }\end{array}$ & 188 & $2.858,52$ \\
\hline 6 & $\begin{array}{l}\text { Gotuzzo, } \\
\text { Eduardo }\end{array}$ & & $\begin{array}{l}\text { Universidad Peruana } \\
\text { Cayetano Heredia/Perú }\end{array}$ & $\begin{array}{l}\text { Enfermedades infecciosas/ } \\
\text { Medicina Tropical }\end{array}$ & 206 & $2.292,87$ \\
\hline 7 & $\begin{array}{l}\text { Malhi, } \\
\text { Yadvinder }\end{array}$ & \multirow{3}{*}{ Azul } & $\begin{array}{l}\text { University of Oxford/ } \\
\text { Inglaterra }\end{array}$ & Ecología/Ciencias de las plantas & 72 & $2.343,77$ \\
\hline 8 & $\begin{array}{l}\text { Phillips, } \\
\text { Oliver L. }\end{array}$ & & $\begin{array}{l}\text { University of Leeds/ } \\
\text { Inglaterra }\end{array}$ & Ecología/Ciencias de las plantas & 40 & $1.697,91$ \\
\hline 9 & Meir, Patrick & & $\begin{array}{l}\text { AustralianNational } \\
\text { University/Australia }\end{array}$ & Ecología/Ciencias de las plantas & 36 & $1.470,17$ \\
\hline 10 & $\begin{array}{l}\text { Kochel, } \\
\text { Tadeusz }\end{array}$ & \multirow{3}{*}{ Amarillo } & $\begin{array}{l}\text { US Naval Medical Research } \\
\text { Center/USA }\end{array}$ & $\begin{array}{l}\text { Medicina Tropical/Salud } \\
\text { ocupacional ambiental pública }\end{array}$ & 112 & $2.531,16$ \\
\hline 11 & Halsey, Eric S. & & $\begin{array}{l}\text { US Naval Medical Research } \\
\text { Center/USA }\end{array}$ & $\begin{array}{l}\text { Medicina Tropical/Enfermedades } \\
\text { infecciosas }\end{array}$ & 82 & $2.158,6$ \\
\hline 12 & $\begin{array}{l}\text { Morrison, } \\
\text { Amy C. }\end{array}$ & & $\begin{array}{l}\text { US Naval Medical Research } \\
\text { Center/USA }\end{array}$ & $\begin{array}{l}\text { Medicina Tropical/Salud } \\
\text { ocupacional ambiental pública }\end{array}$ & 67 & $2.028,11$ \\
\hline 13 & Felix, J. & \multirow{3}{*}{ Morado } & $\begin{array}{l}\text { Fermi National Accelerator } \\
\text { Laboratory/USA }\end{array}$ & $\begin{array}{l}\text { Campos de partículas físicas/ } \\
\text { Astronomía astrofísica }\end{array}$ & 31 & $1.401,95$ \\
\hline 14 & Fields, L. & & $\begin{array}{l}\text { Fermi National Accelerator } \\
\text { Laboratory/USA }\end{array}$ & $\begin{array}{l}\text { Campos de partículas físicas/ } \\
\text { Astronomía astrofísica }\end{array}$ & 31 & $1.401,95$ \\
\hline 15 & Gran, R. & & $\begin{array}{l}\text { University of Minnesota } \\
\text { Duluth/USA }\end{array}$ & $\begin{array}{l}\text { Campos de partículas físicas/ } \\
\text { Astronomía astrofísica }\end{array}$ & 31 & $1.401,95$ \\
\hline
\end{tabular}

$\mathrm{ND}=$ número de documentos; FET = fuerza de enlace total

3.3.2. Acoplamiento bibliográfico de autores en documentos con al menos una afiliación ecuatoriana

En esa misma línea, en la visualización de la red bibliométrica presentada en el Gráfico 7, los círculos representan la magnitud de la fuerza de enlace de acoplamiento bibliográfico de los investigadores. Cuanto más cerca se encuentran dos investigadores en la visualización, mayor será la relación entre ellos según el acoplamiento bibliográfico. Es decir, investigadores que se ubican cerca el uno del otro, tienden a citar las mismas publicaciones, tal es el caso de Prewitt, M. y Abazov, V.M. En general; en los tres clústeres no se muestra un liderazgo de investigadores en la red de acoplamiento bibliográfico. 
Gráfico 7. Visualización de una red de acoplamiento bibliográfico de autores que publicaron en documentos con al menos una afiliación ecuatoriana

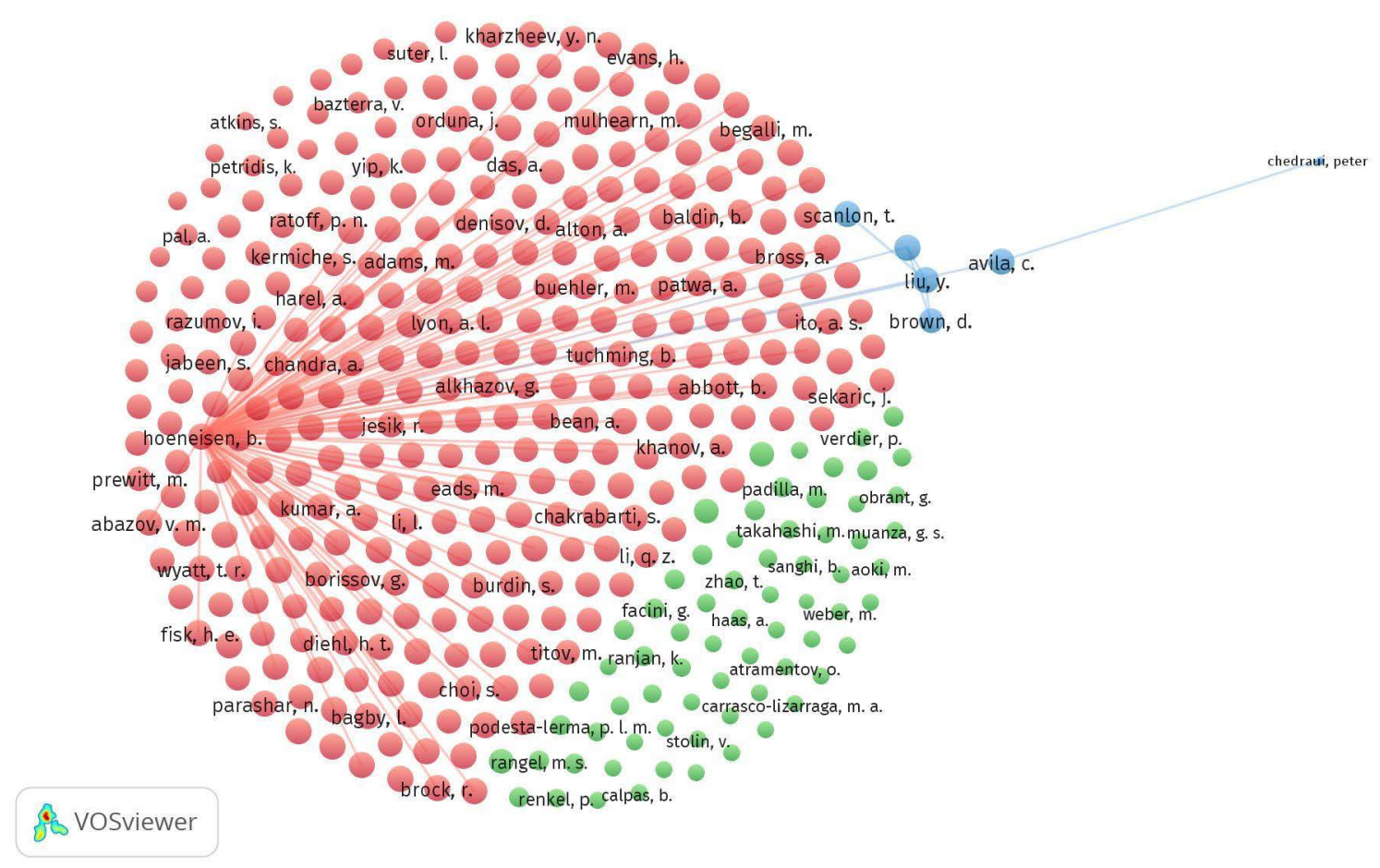

Nota: Se usaron documentos con 500 autores como máximo por documento, autores con 100 documentos como mínimo. De los 50.079 autores, 400 cumplen el umbral; atracción: 2; repulsión: o; resolución de agrupamiento: 1,0

La Tabla 3 muestra la distribución de los autores con mayor fuerza de enlace de acoplamiento bibliográfico en la literatura científica ecuatoriana que pertenecen a los tres clústeres. Se identifica a cada autor con su afiliación, el país de la institución, sus principales áreas de investigación, el número de documentos en el período analizado y la fuerza de enlace total. Un fenómeno particular que se observa es que los investigadores de todos los clústeres investigan sobre campos de partículas física, astronomía y astrofísica. Tienen similar comportamiento en sus fuerzas de enlace y tienden a escribir entre ellos en coautoría, con mucha cantidad de autores por documento. Esa es la razón de tender a citar los mismos documentos en sus investigaciones y por tanto están más acoplados bibliográficamente. Hoeneisen, B., como único autor con afiliación a una institución ecuatoriana que se muestra en la Tabla 3, es el investigador activo con mayor influencia en la actividad investigativa ecuatoriana. 
Tabla 3. Autores de los tres clústeres con mayor fuerza de acoplamiento bibliográfico en documentos con afiliación ecuatoriana

\begin{tabular}{|c|c|c|c|c|c|c|}
\hline$N^{\circ}$ & Autor & Cluster & Institución/país & $\begin{array}{l}\text { Principales áreas temáticas/ } \\
\text { categorías de WoS }\end{array}$ & ND & FET \\
\hline 1 & Hoeneisen, B. & \multirow{5}{*}{ Rojo } & $\begin{array}{l}\text { Universidad San Francisco } \\
\text { de Quito/Ecuador }\end{array}$ & $\begin{array}{l}\text { Campos de partículas físicas/ } \\
\text { Astronomía astrofísica }\end{array}$ & 199 & $6.883,61$ \\
\hline 2 & $\begin{array}{l}\text { Borissov, } \\
\text { Guennadi }\end{array}$ & & $\begin{array}{l}\text { Lancaster University/ } \\
\text { Inglaterra }\end{array}$ & $\begin{array}{l}\text { Campos de partículas físicas/ } \\
\text { Astronomía astrofísica }\end{array}$ & 197 & $6.864,73$ \\
\hline 3 & Abazov, V. M. & & $\begin{array}{l}\text { Joint Institute for Nuclear } \\
\text { Research/Rusia }\end{array}$ & $\begin{array}{l}\text { Campos de partículas físicas/ } \\
\text { Astronomía astrofísica }\end{array}$ & 196 & $6.844,81$ \\
\hline 4 & Abbott, B.S. & & $\begin{array}{l}\text { University of Oklahoma } \\
\text { System/USA }\end{array}$ & $\begin{array}{l}\text { Campos de partículas físicas/ } \\
\text { Astronomía astrofísica }\end{array}$ & 196 & $6.844,81$ \\
\hline 5 & Adams, M.R. & & $\begin{array}{l}\text { University of Illinois } \\
\text { Chicago/USA }\end{array}$ & $\begin{array}{l}\text { Campos de partículas físicas/ } \\
\text { Astronomía astrofísica }\end{array}$ & 196 & $6.844,81$ \\
\hline 6 & $\begin{array}{l}\text { Gruenendahl, } \\
\text { Stefan }\end{array}$ & \multirow{5}{*}{ Verde } & $\begin{array}{l}\text { Fermi National Accelerator } \\
\text { Laboratory/USA }\end{array}$ & $\begin{array}{l}\text { Campos de partículas físicas/ } \\
\text { Astronomía astrofísica }\end{array}$ & 182 & $6.365,39$ \\
\hline 7 & $\begin{array}{l}\text { Gruenewald, } \\
\text { M.W. }\end{array}$ & & $\begin{array}{l}\text { University CollegeDublin/ } \\
\text { Irlanda }\end{array}$ & $\begin{array}{l}\text { Campos de partículas físicas/ } \\
\text { Astronomía astrofísica }\end{array}$ & 180 & $6.278,54$ \\
\hline 8 & $\begin{array}{l}\text { Soeldner- } \\
\text { Rembold, Stefan }\end{array}$ & & $\begin{array}{l}\text { University of Manchester/ } \\
\text { Inglaterra }\end{array}$ & $\begin{array}{l}\text { Campos de partículas físicas/ } \\
\text { Astronomía astrofísica }\end{array}$ & 175 & $6.190,94$ \\
\hline 9 & Padilla, Melissa & & $\begin{array}{l}\text { University of California } \\
\text { Riverside/USA }\end{array}$ & $\begin{array}{l}\text { Campos de partículas físicas/ } \\
\text { Astronomía astrofísica }\end{array}$ & 141 & $4.887,11$ \\
\hline 10 & White, A. & & $\begin{array}{l}\text { University of Texas } \\
\text { Arlington/USA }\end{array}$ & $\begin{array}{l}\text { Campos de partículas físicas/ } \\
\text { Astronomía astrofísica }\end{array}$ & 141 & $4.887,11$ \\
\hline 11 & Liu, Y.F. & \multirow{5}{*}{ Azul } & $\begin{array}{l}\text { NationalTaiwan University/ } \\
\text { Taiwan }\end{array}$ & $\begin{array}{l}\text { Campos de partículas físicas/ } \\
\text { Astronomía astrofísica }\end{array}$ & 199 & $7.047,81$ \\
\hline 12 & Scanlon, Tim & & $\begin{array}{l}\text { University College London/ } \\
\text { Inglaterra }\end{array}$ & $\begin{array}{l}\text { Campos de partículas físicas/ } \\
\text { Astronomía astrofísica }\end{array}$ & 197 & $7.022,81$ \\
\hline 13 & Johnson, Marvin & & $\begin{array}{l}\text { Fermi National Accelerator } \\
\text { Laboratory/USA }\end{array}$ & $\begin{array}{l}\text { Campos de partículas físicas/ } \\
\text { Astronomía astrofísica }\end{array}$ & 197 & $6.869,81$ \\
\hline 14 & Avila, Carlos & & $\begin{array}{l}\text { Universidad de los Andes/ } \\
\text { Colombia }\end{array}$ & $\begin{array}{l}\text { Campos de partículas físicas/ } \\
\text { Astronomía astrofísica }\end{array}$ & 197 & $6.868,81$ \\
\hline 15 & Brown, D. & & Universite de Paris/Francia & $\begin{array}{l}\text { Campos de partículas físicas/ } \\
\text { Astronomía astrofísica }\end{array}$ & 195 & $6.768,14$ \\
\hline
\end{tabular}

$\mathrm{ND}$ = número de documentos; FET = fuerza de enlace total

\subsubsection{Cocitación de autores en documentos con al menos una afiliación peruana}

Para complementar el análisis anterior, se hizo un análisis de cocitación de autores. Este análisis permite revelar la existencia de una estructura intelectual entre los autores cocitados; a mayor número de cocitaciones entre dos autores, mayor será la relación entre ellos (Vargas-Quesada y De-Moya-Anegón, 2007; Perianes-Rodríguez, Waltman y Van Eck, 2016). Así, el Gráfico 8 muestra la visualización de la red de cocitación de autores donde cada círculo representa un autor referenciado en la producción científica peruana y el tamaño de un círculo refleja la fuerza de enlace de cocitación. Tal es el caso de la Organización Mundial de la Salud (World Health Organization) y Abelev, B. con 1.673,7 y 932,2 de fuerza de cocitación respectivamente. Es notorio que entre los autores referenciados se encuentran instituciones y/o organizaciones, además de la World Health Organization también se encuentran el Instituto Nacional de Estadística e Informática (INEI) y el Ministerio de Salud, entre otros. Asimismo, 
Gráfico 8. Visualización de la red de cocitación de autores que publicaron en documentos con al menos una afiliación peruana

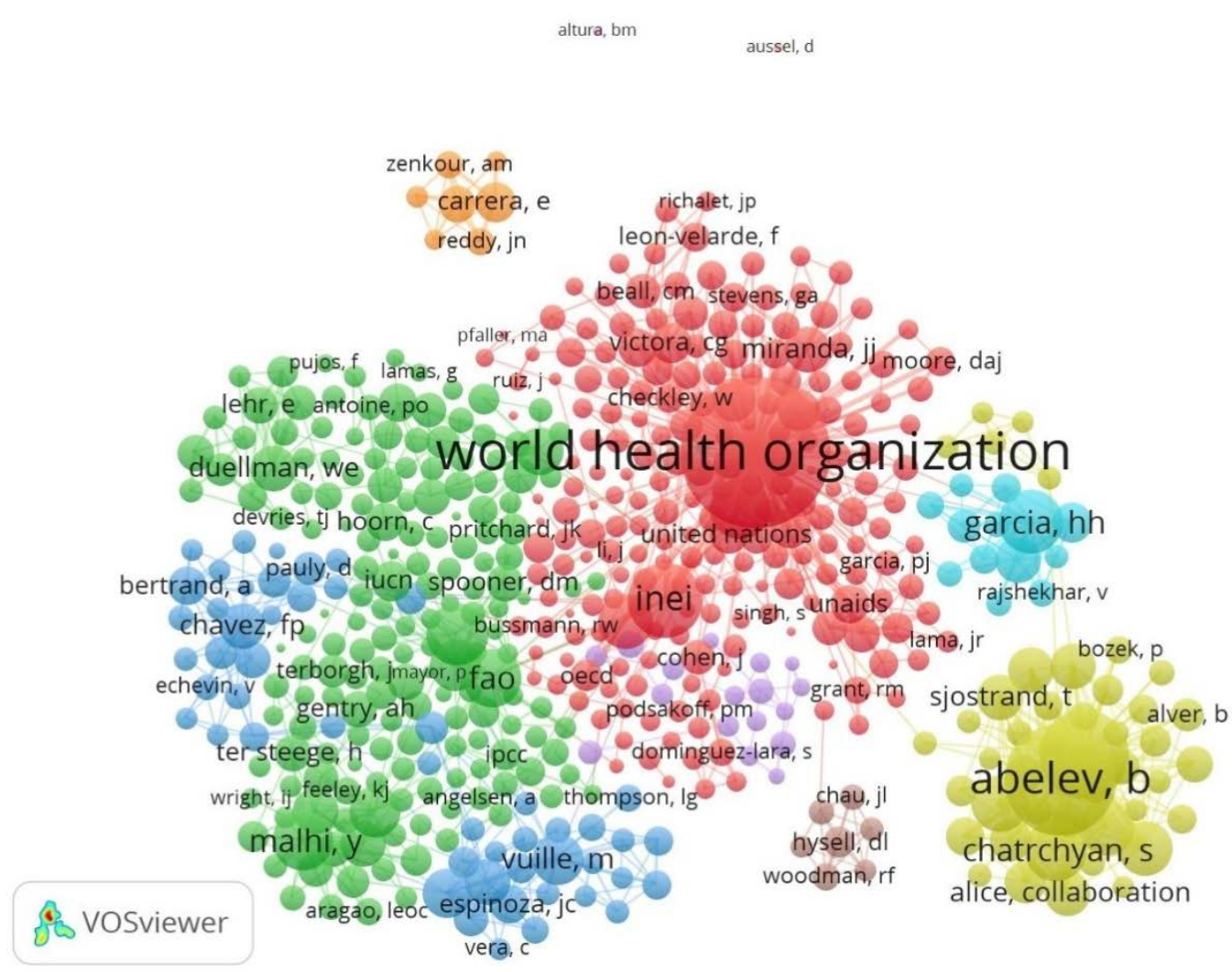

Nota: Se usaron autores con 40 citas como mínimo. De los 251.695 autores, 523 cumplen el umbral; atracción: 2; repulsión: -2; resolución de agrupamiento: 0,5

investigadores referenciados que se encuentran en la visualización cerca el uno del otro, tienden a estar más fuertemente relacionados, basados en cocitaciones, que investigadores ubicados muy lejos el uno del otro. Por ejemplo, Vuille, M. y Espinoza, J.C. tienen una alta relación de cocitación a diferencia de Carrera, E. y Chavez, F.P. que están más distanciados. En la visualización se muestran 10 clústeres o agrupaciones según colores definidos, de las cuales el rojo y el verde son los más grandes.

\subsubsection{Cocitación de autores en documentos con al menos una afliación ecuatoriana}

En el Gráfico 9 cada círculo de la red de visualización bibliométrica representa a un investigador y su tamaño refleja la magnitud de la fuerza de enlace de cocitación de un autor referenciado en la producción científica ecuatoriana. Los autores con mayor fuerza de cocitación son Chatrchyan, S. y Khachatryan, V. con cantidades de 1.501,8 y $1.325,4$ respectivamente. Dado que VOSviewer tiene el enfoque de gráficos basados en distancia, investigadores cerca uno del otro, tienden a estar más fuertemente relaciones según cocitaciones; por ejemplo, Pilato, G. y Meyer, H. A. al igual de los investigadores García, H. H. y Del Brutto, O. H. Existen seis agrupaciones o clústeres según relación de la fuerza de cocitación representados por un color distinto. Se destaca que entre los autores referenciados en la producción científica ecuatoriana también se encuentran instituciones y grupos de colaboración tales como Atlas Collaboration, World Health Organization, Unesco, entre otros. Algunas instituciones como World Health 
Gráfico 9. Visualización de la red de cocitación de autores que publicaron documentos con al menos una afiliación ecuatoriana

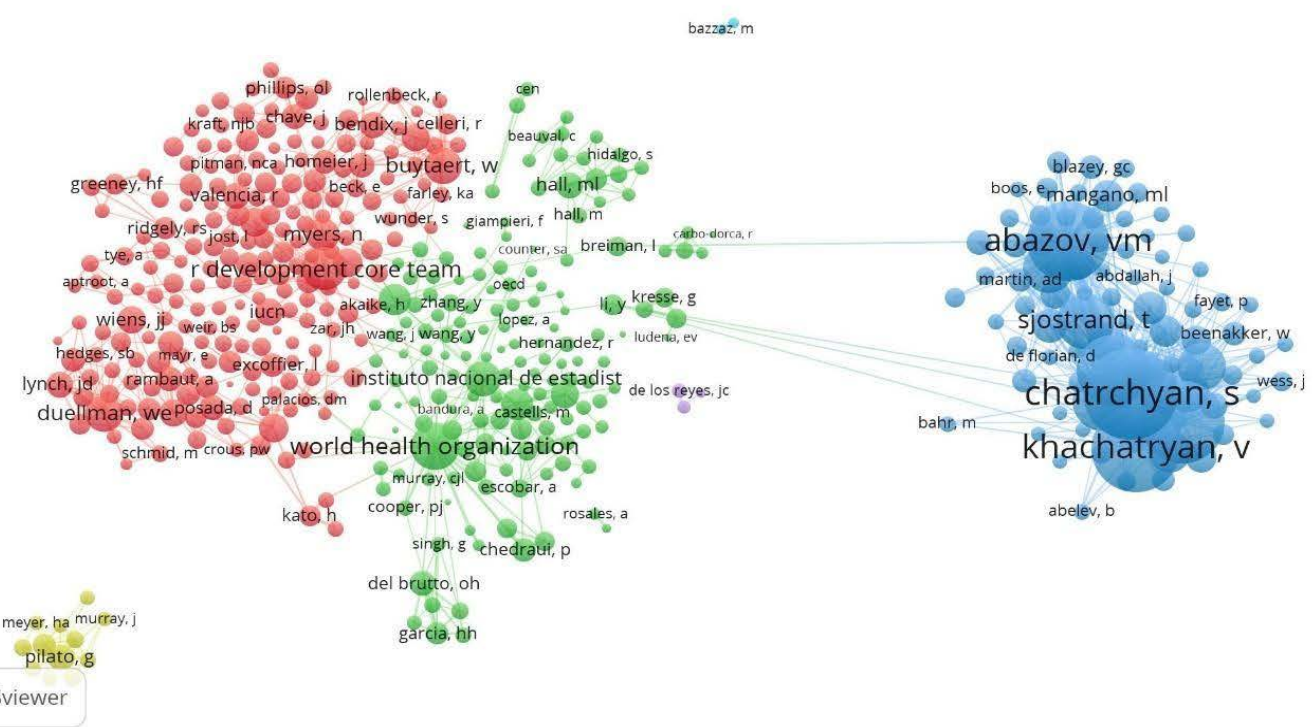

Nota: Se usaron autores con 40 citas como mínimo. De los 243.627 autores, 474 cumplen el umbral; atracción: 2; repulsión: -1; resolución de agrupamiento: 0,5

Organization y $R$ development coreteam y autores como Chatrchyan, S. y García, H. H., se encuentran en los mapas de cocitación tanto de la producción peruana como ecuatoriana (Gráficos 8 y 9) reflejando la importancia de estos en la elaboración de la literatura científica de ambos países.

\section{Discusión y conclusiones}

El estudio de la producción científica de un país es importante, no solo para los investigadores y editores interesados en el tema, sino también para las instituciones que toman decisiones en el gobierno en cuanto al diseño de políticas públicas sobre ciencia y tecnología. Los datos obtenidos con este tipo de estudios son insumos para determinar el estado actual de la investigación científica. Además, son fuentes de información para diseñar estrategias que contribuyan al impulso de la ciencia de un país, una institución, una temática y un investigador. Esta investigación demuestra que la implementación de políticas científicas en Perú y Ecuador han generado productos positivos. Uno de los más importantes, es que ambos han dedicado muchos recursos para mejorar y sostener la cultura de la publicación científica, y cada vez más sus actores han mejorado sus prácticas.

Ambos países evidencian que su producción científica acumulada tiene un crecimiento exponencial en el período analizado. Este notable crecimiento se basa en que las estrategias adoptadas por Perú y Ecuador incentivaron el crecimiento de la producción científica con el incremento del gasto en I+D en el PIB. Ambos países presentaron en el año 2014 una mayor inversión en I+D del PIB en comparación con los años 2012 y 2013. No obstante, se necesita destinar más fondos en gastos en I+D como lo hacen 
países latinoamericanos que tienen mayor producción científica (Brasil, Argentina, México) los cuales destinan por encima del 0,50\%.

Perú en comparación con Ecuador tiene mayor producción científica acumulada en revistas indexadas en WoS durante los años 2009-2018. Sin embargo, Ecuador tiene mayor producción científica que Perú en los tres últimos años (2016, 2017 y 2918). Y, mediante los modelos de regresión cuadráticos estimados, la producción científica de Ecuador tiene un pronóstico de seguir creciendo más que Perú. Esto está relacionado con tres aspectos para Ecuador. Primero, la implementación del escalafón docente e investigador (posiblemente uno de los más competitivos de Latinoamérica); segundo, el programa de becas para estudios doctorales que se mantuvo vigente hasta el año 2018; y, por último, fijó el 6\% como porcentaje mínimo que toda institución de educación superior que recibía dinero del Estado debía asignar de sus presupuestos institucionales solamente en investigación (Ecuador. Presidencia de la República, 2010).

El top cinco de las instituciones más productivas tanto en Perú como en Ecuador son universidades. En Perú destacan la Universidad Peruana Cayetano Heredia (UPCH), la Pontificia Universidad Católica del Perú (PUCP), la Universidad Nacional Agraria La Molina (UNAM), la Universidad Nacional Mayor de San Marcos (UNMSM) y la Universidad Peruana de Ciencias Aplicadas (UPC). En Ecuador, la Universidad San Francisco de Quito (USFQ), la Universidad de Los Andes (UNIANDES), la Universidad de Cuenca (UC), la Pontificia Universidad Católica del Ecuador (PUCE) y la Universidad Técnica Particular de Loja (UTPL). Estos resultados se deben a que en Perú y en Ecuador se implementaron políticas públicas a favor de la investigación científica en universidades públicas y privadas, cambios en las legislaciones y programas que favorecen el crecimiento científico y facilitan la colaboración internacional. También se otorgan incentivos a los docentes e investigadores para estimular la publicación, y fondos destinados a financiar proyectos de investigación nacional e internacional que en mayor medida resuelvan una problemática y tengan un aporte social, con base en los objetivos del desarrollo sostenible.

En ese contexto, en Perú juegan roles importantes la implementación de la Ley $\mathrm{N}^{\circ}$ 30220 (Ley Universitaria), el CONCYTEC y la SUNEDU (Mayta-Tristánet al., 2019), además de la categoría de docente investigador Renacyt. Por parte de Ecuador, la Constitución (artículos 385-388), la LOES, la SENESCYT, el CEAACES, el programa Prometeo "Viejos Sabios" en su momento (Castillo y Powell, 2019), además de un escalafón docente competitivo y el programa de becas para estudios doctorales. Hay que considerar que, en Ecuador, el acceso a las bases de datos de información científica de calidad como es WoS y Scopus por parte de las universidades es aún escasa. Muy pocas tienen el financiamiento necesario para tener dentro de su oferta estos recursos de información, por ende, convierte el esfuerzo ecuatoriano en algo más meritorio, ya que desde el año 2013 las universidades públicas no reciben apoyo financiero para suscribir estos recursos de información científica.

Entre las diez primeras categorías en las que más producción científica registra Perú pertenecen con mayor presencia a las de salud ocupacional y ambiental, enfermedades infecciosas, medicina tropical, las ciencias ambientales y de plantas. Estos resultados concuerdan con las políticas implementadas por el CONCYTEC en el Plan Nacional Estratégico de Ciencia Tecnología e Innovación (2006), en las que las ciencias de la vida y biotecnologías son áreas del conocimiento para atender a los sectores prioritarios. Coinciden también con los resultados presentados por el CONCYTEC (2014) de que el área temática que concentra la mayor parte del esfuerzo investigador es la medicina, con un $44.7 \%$. Asimismo, se debe a que tres de las cinco universidades que más contribuyen a la producción científica (UPCH, UNMSM y UPC) tienen en mayor proporción un perfil disciplinar en el campo de la medicina (Web of Science, 2020). 
Ecuador por su parte, presenta mayor productividad en las categorías de educación, ciencias ambientales, ingeniería y ciencias sociales. Esto se debe a que las áreas priorizadas por el gobierno y atendidas por el SENESCYT mediante los prometeos fueron educación, agricultura, medicina, biología y ciencias del mar. A su vez, para el programa de becas doctorales a las mejores universidades del mundo, también se dieron prioridad a estas áreas. Otro proyecto a destacar fue la creación de 4 universidades emblemáticas financiadas por el Estado: Universidad Yachay (ciudad del conocimiento) ubicada en la provincia de Imbabura, Universidad Regional Amazónica Ikiam ubicada en la provincia de Napo, Universidad de las Artes ubicada en Guayaquil y la Universidad de Educación ubicada en la provincia de Azogues en el austro ecuatoriano. Todos estos eventos se vieron reflejados en el crecimiento exponencial de la producción científica en revistas de alto impacto, y en el fortalecimiento de la colaboración internacional, ya que un objetivo adicional de los prometeos era la de fomentar redes con universidades y grupos de investigación más avanzados.

Se encontró en la producción académica de ambos países que el artículo científico predomina con más del 70\%; esto obedece fundamentalmente a tres factores. Primero, WoS es una base que indexa preferentemente revistas. Segundo, la tendencia en la ciencia moderna es que los científicos difunden los resultados de sus investigaciones en formato artículo, porque la publicación y por ende la difusión es más rápida que en otros formatos como el libro. Tercero, en Perú las áreas de mayor producción son salud ocupacional y ambiental, enfermedades infecciosas y medicina tropical, mientras que en Ecuador son investigación educativa, ecología de ciencias ambientales e ingeniería. La mayoría de los investigadores de estas áreas del conocimiento forman parte de las ciencias duras, donde la preferencia de publicación de los investigadores es el artículo científico. Aunque en Ecuador el área con la mayor producción es investigación educativa, esa misma preferencia en la publicación se presenta en las ciencias sociales.

El análisis de los mapas de visualización de redes bibliométricas basados en vínculos de acoplamiento bibliográfico y de cocitación de autores permiten evidenciar la estructura intelectual de la producción científica peruana y ecuatoriana. En efecto, en el caso peruano se logra identificar a Gilman, R., Miranda, J. y Checkley, W. entre los autores con mayor fuerza de acoplamiento bibliográfico. Los investigadores que pertenecen a los principales clústeres investigan temas del área de salud pública, medicina tropical, enfermedades infecciones, ecología; confirmando así los resultados obtenidos de la producción científica según categorías temáticas. En la producción científica ecuatoriana, el AABA constata que las áreas temáticas con mayor actividad de investigación son campos de partículas físicas, astronomía y astrofísica. A nivel individual, Hoeneisen, B., con afiliación a la Universidad San Francisco de Quito, es uno de los autores con mayor fuerza de acoplamiento bibliográfico que le convierte en el investigador activo con mayor influencia en la actividad científica ecuatoriana.

Los vínculos de cocitación de autores presentan una mirada retrospectiva de los autores y documentos más influyentes en un campo de investigación. Con base a este argumento, el ACA permite identificar comunidades invisibles, mediante la conexión de autores representativos tales como: Abelev, B. la World Health Organization, el Instituto Nacional de Estadística e Informática (INEI), el Ministerio de Salud (MINSA), entre otros en la producción científica peruana. Con esto, no necesariamente las entidades referenciadas son autores individuales, sino que pueden ser instituciones u organizaciones que revelan tener cierta importancia en el proceso de la elaboración de trabajos científicos con afiliación peruana. Ecuador muestra un comportamiento similar, entre los autores con mayor fuerza de cocitación están Chatrchyan, S. y Khachatryan, V. pertenecientes al clúster azul. También se encuentran instituciones como Atlas Collaboration, World Health Organization, Unesco. 
Se demuestra que el papel que cumplen las universidades de ambos países es preponderante para el desarrollo de la investigación y del país. Sin embargo, aún falta mucho que recorrer para estar al nivel de otros países de la región y del norte global. A partir de este trabajo se sugieren dos direcciones principales. Primero, sería importante que los gobiernos y los responsables de gestionar la política científica refuercen la agenda de la producción científica de calidad. Segundo, se recomienda que futuros estudios analicen la producción científica de estos países, mediante los indicadores de impacto, de colaboración y las redes bibliométricas de coautoría atendiendo las demandas institucionales y gubernamentales. Dado que en este trabajo solo se tomaron como fuente de información la plataforma Web of Science, se sugiere que estudios posteriores usen otras bases de datos como: Scopus, Dimensions, Lens, etc. En efecto, cualquiera de estas iniciativas sería una gran adición a este trabajo que permitirían complementar los resultados obtenidos aquí.

El destino de los dos países se dirige hacia un crecimiento integral de sus sistemas universitarios. En el caso de Ecuador la sociedad espera que vuelvan a estar en vigencia los programas de becas para investigadores. En Perú, se espera que los cambios que se están implementando sean sostenidos en el tiempo.

\section{Agradecimientos}

A los grupos de investigación EILA y CIGETMEN de la Universidad Nacional Mayor de San Marcos; a la Dirección de Investigación de la Universidad Peruana de Ciencias Aplicadas. Asimismo, a Ludo Waltman del CWTS Leiden University, The Netherlands y a Salvador E. Vázquez de la Universidad Nacional Autónoma de México por sus comentarios muy útiles al texto preliminar. 


\section{Q Referencias bibliográficas}

》Álvarez-Muñoz, Patricio y Mario Pérez-Montoro. 2015. Análisis de la producción y de la visibilidad científica de Ecuador en el contexto andino (2000-2013). En El Profesional de la Información. Vol. 24, no. 5, 577-586. <https://doi.org/10.3145/ epi.2015.sep.07>

" Amaya, Elard; Benoit Mougenot y Percy Herrera-Añazco. 2019. Gender disparities in scientific production: A nationwide assessment among physicians in Peru. En PLoS ONE. Vol. 14, no. 11. <https://doi.org/10.1371/journal.pone.0224629>

»Asmat-Vega, Nicanor; César Borja-Villanueva; Luis Bernuy-Torres; Danny Lizarzaburu Aguinaga y John Morillo-Flores. 2019. Estudio bibliométrico de la producción científica sobre TIC en Perú (2010-2017). En Propósitos y Representaciones. Revista de Psicología Educativa. Vol. 7, no. 2, 196-209. <http://dx.doi. org/10.20511/pyr2019.v7n2.269>

»Banco Mundial. 2019. Gasto en investigación y desarrollo (\% del PIB). <https:// datos.bancomundial.org/indicador/GB.XPD.RSDV.GD.ZS?end=2017\&most_ recent_year_desc=true\&start=1996\&type=shaded\&view=map $>$ [Consulta: 24 febrero 2020].

" Bazm, Soheila; Reihaneh Bazm y Farzaneh Sardari. 2019. Growth of health literacy research activity in three Middle Eastern countries. En BMJ Health and Care Informatics. Vol. 26, no. 1, 1-6. <https://doi.org/10.1136/bmjhci-2019-000027>

"Bornmann, Lutz y Hans-Dieter Daniel. 2008. What do citation counts measure? A review of studies on citing behavior. En Journal of Documentation. Vol. 64, no. 1, 45-80. <https://doi.org/10.1108/00220410810844150>

"Carvajal-Tapia, Aarón y Eduardo Carvajal-Rodríguez. 2019. Producción científica en ciencias de la salud en los países de América Latina, 2006-2015: análisis a partir de Scielo. En Revista Interamericana de Bibliotecología. Vol. 42, no. 1, 15-21. <https://doi.org/10.17533/udea.rib.v42n1ao2>

»Castillo, José-Antonio y Michael Powell. 2019. Análisis de la producción científica del Ecuador e impacto de la colaboración internacional en el periodo 2006-2015. En Revista Española de Documentación Científica. Vol. 42, no. 1, 1-16. <https://doi.org/10.3989/redc.2019.1.1567>

"CEAACES Consejo de Evaluación, Acreditación y Aseguramiento de la Calidad de la Educación Superior. 2018. Modelo de evaluación institucional de universidades y escuelas politécnicas 2018. <https://www.ucsg.edu.ec/wp-content/uploads/ transparencia/Modelo-evaluacion-preliminar-universidades-escuelas-politecnicas2018.pdf> [Consulta: 6 diciembre 2019].

» CONCYTEC. 2006. Plan nacional estratégico de ciencia, tecnología e innovación para la competitividad y el desarrollo humano PNCTI 2006 - 2021. Lima: CONCYTEC.

»CONCYTEC. 2014. Principales indicadores bibliométricos de la actividad científica peruana, 2006-2011. Lima: CONCYTEC.

»Ecuador. Presidencia de la República. 2010. Ley orgánica de educación superior. En Registro oficial. Órgano del gobierno de Ecuador. Año 2, no. 298, 12 de octubre. <https://spryn.finanzas.gob.ec/esipren-web/archivos_html/file/Ley\%20 Org\% 3 \%A1nica\%20de\%20Educacion\%20Superior\%202010.pdf> [Consulta: 6 agosto 2020]. 
» Gago Medina, Alberto. 2016. Sobre la Importancia de la Investigación Básica. En Pedraglio Mendoza, Santiago, coord. Aproximaciones a la Educación Universitaria. Lima: Pontificia Universidad Católica del Perú. p. 69-77. <https://s3.amazonaws. com/files.pucp.edu.pe/puntoedu/wp-content/uploads/2016/o6/Aproximacimaciones-a-la-educacion-universitaria.pdf> [Consulta: 3 diciembre 2019].

» Huamaní, Charles y Percy Mayta-Tristán. 2010. Producción científica peruana en medicina y redes de colaboración, análisis del Science Citation Index 20002009. En Revista Peruana de Medicina Experimental y Salud Pública, Vol. 27, no 3 , 315-325. <https://doi.org/10.1590/S1726-46342010000300003>

» Jaramillo-Salazar, Hernán; María Alejandra Botiva y Andrés Zambrano. 2004. Políticas y resultados de ciencia y tecnología en Colombia. Bogotá: Centro Editorial Universidad del Rosario.

»Kessler, Michael M. 1963. Bibliographic coupling between scientific papers. En JASIST Journal of the Association for Information Science and Technology. Vol. 14, no. 1, 10-25. <https://doi.org/10.1002/asi.5090140103>

» Lascurain-Sánchez, MaríaLuisa. 2006. La evaluación de la actividad científica mediante indicadores bibliométricos. En Bibliotecas. Vol. 24, no. 1-2, 9-26. <https:// www.revistas.una.ac.cr/index.php/bibliotecas/article/view/429> [Consulta: 7 enero 2020].

"Leydesdorff, Loet. 2008. Caveats for the use of citation indicators in research and journal evaluations. En Journal of the American Society for Information Science and Technology. Vol. 59, no. 2, 278-287. <https://doi.org/10.1002/asi.20743>

» Mayta-Tristán, Percy; Carlos J. Toro-Huamanchumo; Joel Alhuay-Quispe y Josmel Pacheco-Mendoza. 2019. Producción científica y licenciamiento de escuelas de medicina en el Perú. En Revista Peruana de Medicina Experimental y Salud Pública. Vol. 36, no. 1, 106-115. <https://doi.org/10.17843/rpmesp.2019.361.4315>

»Moed, Henk F. 2009. New developments in the use of citation analysis in research evaluation. En Archivumim munologiae et therapiae experimentalis. Vol. 57, no. 13, 13-18. <https://doi.org/10.1007/s00005-009-0001-5>

" Ocaña-Fernández, Yolvi; Isabel Menacho Vargas; Luis Nuñez Lira; Flor de María Sánchez Aguirre; Rafael Garay-Argandoña; Ronald M. Hernández y Karel Llopiz Guerra. 2019. Peruvian scientific production on mental health in Scopus, 2010-2018. En International Journal of Psychosocial Rehabilitation. Vol. 23, no. 1. <https://siis.unmsm.edu.pe/es/publications/peruvian-scientific-production-on-mental-health-in-scopus-2010-20-2> [Consulta: 27 enero 2020].

»Perianes-Rodríguez, Antonio; Ludo Waltman y Nees-Jan Van Eck. 2016. Constructing bibliometric networks: A comparison between full and fractional counting. En Journal of Informetrics. Vol. 10, no. 4, 1178-1195. <https://doi. org/10.1016/j.joi.2016.10.006>

»Perú. Congreso de la República. 2014. Ley Universitaria. Ley no. 30220. En El Peruano, Año 31, no. 12914, 9 de julio. <https://www.sunedu.gob.pe/wp-content/ uploads/2017/04/Ley-universitaria-30220.pdf> [Consulta: 28 diciembre 2019].

"Pouris, Anastassios. 2012. Scientometric research in South Africa and successful policy instruments. En Scientometrics. Vol. 91, no. 2, 317-325. <https://doi. org/10.1007/s11192-011-0581-9>

"Price, Derek J. de Solla. 1963. Little science, big science. New York, NY: Columbia University Press. <https://doi.org/10.7312/pric91844>

»Rivera García, Christian Geovanny; Julia M. Espinosa Manfugás y Yulima D. Valdés Bencomo. 2017. La investigación científica en las universidades ecuatorianas: 
Prioridad del sistema educativo vigente. En Revista Cubana de Educación Superior. Vol. 36, no. 2, 113-125. <http://scielo.sld.cu/scielo.php?script=sci_arttext\&p id=So257-43142017000200011> [Consulta: 24 enero 2020].

»Roman-Gonzalez, Avid; Antony Ciriaco-Susanibar y Natalia I. Vargas-Cuentas. 2019. Comparing the scientific production of Peruvian universities with equitable indexes. En Advances in Science, Technology and Engineering Systems. Vol. 4, no. 4, 394-403. <https://doi.org/10.25046/ajo40448>

»Romaní, Franco y César Cabezas. 2018. Indicadores bibliométricos de las publicaciones científicas de la Revista Peruana de Medicina Experimental y Salud Pública, 2010-2017. En Revista Peruana de Medicina Experimental y Salud Pública. Vol. 35, no. 4, 620-629. <https://doi.org/10.17843/rpmesp.2018.354.3817>

»Roy, Sanku-Bilas. 2019. Research output of biological science during 1901-1945: A scientometric analysis. En DESIDOC Journal of Library and Information Technology. Vol. 39, no. 3, 96-103. <https://doi.org/10.14429/djlit.39.3.14065>

"Sisa, Iván; Mauricio Espinel; Marco Fornasini y Gonzalo Mantilla. 2011. La producción científica en ciencias de la salud en Ecuador. En Revista Panamericana de Salud Pública. Vol. 30, no. 4, 388-92. <https://www.scielosp.org/article/ rpsp/2011.v30n4/388-392/> [Consulta: 4 febrero 2020].

»SUNEDU Superintendencia Nacional de Educación Superior Universitaria. 2018. Informe bienal sobre la realidad universitaria peruana. <https://www.sunedu.gob. pe/informe-bienal-sobre-realidad-universitaria/> [Consulta: 13 diciembre 2019].

»Urbizagástegui-Alvarado, Rubén. 2014. La bibliometría en el Perú. En Letras. Revista de investigación de la Facultad de Letras y Ciencias Humanas. Vol. 85, no. 122, 247-269. <http://revista.letras.unmsm.edu.pe/index.php/le/article/view/269> [Consulta: 24 enero 2020].

»Van Eck, Nees-Jan y Ludo Waltman. 2014. Visualizing bibliometric networks. En Ding, Ying; Ronald Rousseau y Dietmar Wolfram, eds. Measuring scholarly impact: Methods and practice. Springer, p. 285-320. <https://doi.or g/10.1007/978-3-319-10377-8_13>

»Vargas-Quesada, Benjamín y Félix de-Moya-Anegón. 2007. Visualizing the structure of science. New York, NY: Springer. <https://doi.org/10.1007/3-540-69728-4>

»Wang, Yuan; Nan Lai; Jian Zuo; Guanyi Chen y Huibin Du. 2016. Characteristics and trends of research on waste-to-energy incineration: A bibliometric analysis, 1999-2015. En Renewable and Sustainable Energy Reviews. Vol. 66, 95-104. <https://doi.org/10.1016/j.rser.2016.07.006>

"Web of Science. 2020. Web of Science Core Collection. <https://webofscience. com> [Consulta: 3 agosto 2020].

»White, Howard D. y Belver C. Griffith. 1981. Author cocitation: A literature measure of intellectual structure. En Journal of the American Society for Information Science. Vol. 32, no. 3, 163-171. <https://doi.org/10.1002/asi.4630320302>

»Yasar-Akcali, Burcay y Elcin Sismanoglu. 2015. Innovation and the effect of research and development (R\&D) expenditure on growth in some developing and developed countries. En Procedia-Social and Behavioral Sciences. Vol. 195, 768775. <https://doi.org/10.1016/j.sbspro.2015.06.474>

"Zhao, Dangzhi y Andreas Strotmann. 2008. Evolution of Research Activities and Intellectual Influences in Information Science 1996-2005: Introducing Author Bibliographic-Coupling Analysis. En Journal of the American Society for Information Science and Technology. Vol. 59, no. 13, 2070-2086. <https://doi.org/10.1002/ asi.20910> 